\title{
Revisión
}

\section{Estatinas en la insuficiencia cardiaca}

(Statins in Heart Failure)

Allan Ramos-Esquivel

Resumen

Desde su descubrimiento hace cerca de 30 años, las estatinas han desempeñado un papel importante en el tratamiento y prevención de las enfermedades cardiovasculares, con base, inicialmente, en las propiedades hipolipemiantes de esta clase de fármacos. Con el advenimiento de nuevos conocimientos sobre su mecanismo de acción y de sus propiedades pleiotrópicas, se ha planteado la utilidad clínica de las estatinas en diversas enfermedades. Ha surgido evidencia sugestiva de la eficacia de estos fármacos en la insuficiencia cardiaca a partir de múltiples teorías. A continuación se revisan el fundamento fisiopatológico que sustenta su utilización en este síndrome y la actual evidencia clínica en favor o en contra de tal uso.

Descriptores: insuficiencia cardiaca, estatinas

\section{Abstract}

Since their discovery 30 years ago, statins have played an important role in the treatment and prevention of cardiovascular diseases based on their lipid lowering properties. Given their pleiotropic activity, their clinical utility in the treatment of several diseases has been discussed. Lately, based on multiple theories, evidence has emerged regarding the efficacy of these drugs in heart failure patients. A review is presented of the physiopathological basis supporting their use and of clinical evidence in favor and against it.

Key words: heart failure, statins

Recibido: 18 de agosto de 2008 Aceptado: 20 de enero de 2009

La utilidad de las estatinas es bien reconocida en los pacientes con dislipidemia y factores de

Departamento de Farmacología. Universidad de Costa Rica. Programa de Maestría en Ciencias Biomédicas. Universidad de Costa Rica

Abreviaturas: $\mathrm{FE}$, fracción de eyección; GTPasa, guanosín trifosfatasa; IC, índice de confianza; ICC, insuficiencia cardiaca congestiva.

\section{Correspondencia}

Allan Ramos Esquivel Email:allan.ramos@ucr.ac.cr Dirección: Heredia Centro. Av 4 Calles 5 \& 7. esgo cardiovascular, y existen múltiples estudios de alta evidencia clínica que apoyan su uso en la prevención primaria y secundaria de eventos cardiovasculares. ${ }^{1,2}$

No obstante, el empleo de estos fármacos en pacientes con insuficiencia cardiaca ya establecida, como un medicamento adicional que proporcione una mejoría en la morbimortalidad de los pacientes, es un tema de controversia, fundamentalmente debido a que la mayoría de estudios con estatinas han excluido a quienes padecen este síndrome. ${ }^{3}$

A continuación se exponen las bases fisiopatológicas y farmacológicas que suponen la eficacia de este grupo de fármacos, así como los resultados principales de algunos ensayos clínicos agrupados según la metodología del estudio. 
La insuficiencia cardiaca congestiva (ICC) es una condición que afecta en Estados Unidos a 5 millones de personas anualmente; se diagnostican más de medio millón de casos nuevos por año, y se ha convertido, además, en la principal causa de hospitalizaciones en ese país. ${ }^{4}$

A pesar del advenimiento de nuevas terapias para el control de la ICC, todavía persiste la misma mortalidad reportada hace más de 25 años. ${ }^{5}$ Asimismo, con los cambios en el estilo de vida de la sociedad actual, se espera que la cifra de pacientes con ICC aumente, conforme avanzan también la enfermedad arterial coronaria y la hipertensión arterial; principales factores de riesgo para esta patología.

\section{Fisiopatología de la ICC y pleiotropismo de las estatinas}

La insuficiencia cardíaca se caracteriza por la presencia de respuestas neurohormonales compensadoras, las cuales, teleológicamente, persiguen mantener una adecuada capacidad contráctil del miocito cardiaco. Así, por ejemplo, la actividad aumentada del sistema renina - angiotensina aldosterona y el mayor tono neuronal simpático, garantizan una correcta redistribución del flujo sanguíneo y una elevación del gasto cardíaco, ${ }^{6}$ que eventualmente llevará a un círculo vicioso de perpetuación de las anormalidades hemodinámicas que predisponen a la ICC.

Una de las conocidas respuestas adaptativas que surgen en este síndrome es la hipertrofia ventricular, que inicialmente disminuye el estrés parietal, pero implica una mayor resistencia al llenado ventricular y un aumento de las demandas metabólicas del tejido miocárdico. Este proceso es mediado, al menos en parte, por la acción de las proteínas GTPasas pequeñas, tales como Rho y Rac1. ${ }^{7,8}$ Más aun, la hipertrofia inducida por la epinefrina, la endotelina, la angiotensina - II, los radicales libres ${ }^{9,10}$ o por la hipertensión arterial, ${ }^{11}$ es mediada, al menos en parte, por este tipo de proteínas. ${ }^{12}$ Similarmente, el estrés oxidativo, consecuencia de la alteración neurohormonal descrita, constituye un potente estimulante de la remodelación cardíaca. ${ }^{13,14} \mathrm{De}$ hecho, la existencia de especies reactivas del oxígeno induce a la hipertrofia ventricular y promueve la apoptosis de los cardiomiocitos, ${ }^{13}$ disminuyendo la cantidad de adenosín trifosfato disponible para cada contracción y favoreciendo la expresión de genes relacionados con la alteración contráctil. $^{15,16}$ Además, los radicales libres potencian la proliferación de los fibroblastos cardíacos, propiciando la remodelación de la matriz extracelular ${ }^{17,18}$ y la alteración de la función endotelial. ${ }^{19-21}$

Al conocer las propiedades pleiotrópicas de las estatinas, es decir, aquellas más allá de disminuir las cifras de colesterol sanguíneo, ${ }^{23}$ es fácil comprender por qué utilizar tales fármacos en la ICC, ya que al inhibir la 3-OH-3-metilglutaril coenzima A reductasa, se disminuyen también las concentraciones intracelulares del mevalonato y de los isoprenoides; mediadores químicos fundamentales para la activación de las proteínas GTPasas pequeñas. ${ }^{23}$ Además, existe evidencia de las propiedades antioxidantes de esta clase de fármacos, ya que promueven la actividad catalítica de enzimas que disminuyen el estrés oxidativo e interfieren en la propiedad oxidativa de la angiotensina II. ${ }^{24-28}$ Más aun, las estatinas, en el contexto de la ICC, parecen modular la marcada actividad simpática en modelos experimentales de esta condición, ${ }^{29-31}$ a la vez que conducen a una disminución de marcadores inflamatorios y sustancias proagregantes (disminución de la proteína $\mathrm{C}$ reactiva, antitrombina III, factor $\mathrm{V}$, activador del plasminógeno tisular, entre otros), ${ }^{32-34}$ mejoran el balance neurohormonal y restablecen la función endotelial. ${ }^{35-41}$

Con esta base teórica tan sugestiva, se iniciaron diversos estudios ex vivo que corroboraron experimentalmente la mejoría en diversos parámetros y modelos de insuficiencia cardíaca y de hipertrofia ventricular. Así se comprobó la prevención de la hipertrofia cardíaca y la mejoría de la función cardiovascular con el uso de estatinas en animales de experimentación, ${ }^{42,}{ }^{43}$ sugiriendo que su empleo en humanos podría retardar la progresión de la insuficiencia cardíaca hacia estadios refractarios al tratamiento usual.

Dos análisis post hoc de estudios aleatorizados con el uso de estatinas, fueron los que inicialmente introdujeron una virtual eficacia de las estatinas en pacientes con ICC. El estudio $4 \mathrm{~S}$, que comparó la eficacia de simvastatina versus placebo, excluyó en un principio a pacientes con ICC sintomática de base, pero en un análisis del seguimiento de los pacientes que desarrollaron ICC a lo largo del estudio $(n=184$ en el grupo de simvastatina $y=228$ en el grupo placebo), se demostró que quienes utilizaron la estatina presentaban una mortalidad menor a los 5 años, que quienes recibieron placebo $\left(25.5 \%\right.$ versus $31.9 \%$, respectivamente). ${ }^{44}$ El estudio CARE comparó la eficacia de la pravastatina versus el placebo y evidenció también en el subgrupo de pacientes con una F.E. menor al 40\%, una reducción significativa del riesgo relativo de un evento cardiovascular $\operatorname{del} 28 \%(0.04-0.45$ IC: $95 \%){ }^{45}$

Cabe señalar que en ambos estudios las aseveraciones hechas con respecto al grupo de pacientes con ICC, se sometieron al análisis de subgrupos y en forma retrospectiva, con la subsecuente limitante estadística.

\section{Estudios retrospectivos de cohortes en pacientes con ICC}

Diversos estudios retrospectivos han señalado la probable utilidad de las estatinas en pacientes con ICC establecida, tanto isquémica como no isquémica. Así, por ejemplo, se ha señalado que aquellos pacientes con una $\mathrm{FE}$ menor al $40 \%$ tratados con estos medicamentos, mostraron una mayor sobrevida libre de trasplante cardiaco que el grupo que no los usaba, con una reducción del riesgo absoluto cercano al $20 \%$, y una reducción del riesgo relativo del 59\% (HR: 0.41 IC 95\%=0.18-0.94). ${ }^{46}$ 
Un análisis llevado a cabo en pacientes de 66 a 85 años, estableció, por su parte, una reducción significativa del $28 \%$ en el riesgo relativo del objetivo primario compuesto (mortalidad de cualquier causa, infarto agudo de miocardio no fatal o evento cerebrovascular no fatal) $(95 \% \mathrm{IC}=0.63$ 0.83 ) en los pacientes que usaban estatinas. ${ }^{47}$

Otro estudio de cohortes ( $\mathrm{n}=24$ 598), demostró, por su parte, que pacientes con ICC que iniciaron terapia con estatinas, presentaban una reducción del $24 \%$ y del $21 \%$ del riesgo relativo de mortalidad y hospitalización por ICC, respectivamente. ${ }^{49}$ Un reciente estudio observacional mostró, tras el análisis retrospectivo de 54690 pacientes mayores de 65 años con ICC, que el grupo de pacientes que recibieron estatinas después del diagnóstico (20\%), presentaban una sobrevida mayor al año y a los 3 años que el resto de pacientes $(\mathrm{HR}=0.80,95 \%$ IC $=0.76-0.84$ y $\mathrm{HR} 0.82,95 \% \mathrm{IC}=0.79$ 0.85 , respectivamente). ${ }^{49}$

\section{Estudio prospectivo de cohortes en pacientes con ICC}

Un análisis secundario de un estudio de 6427 pacientes portadores de ICC con enfermedad arterial coronaria establecida y con nefropatía, mostró que quienes utilizaron estatinas $(40 \%$ del total) durante el período de estudio, presentaban una reducción significativa del riesgo relativo de muerte por cualquier causa, del $21 \%$. (CI 95\%: IC $=0.64$ $-0.97)^{50}$

\section{Estudios retrospectivos de pacientes con ICC que utilizaron estatinas}

Un análisis retrospectivo del estudio PRAISE (diseñado para evaluar la mortalidad con el uso de amlodipina versus placebo en pacientes con ICC sistólica), evidenció una reducción del riesgo relativo del $62 \%$ en la mortalidad de los 134 pacientes que utilizaron estatinas, durante las 15 semanas de seguimiento, logrando prevenir una muerte por cada 5 pacientes tratados en un año (HR=0.38, 95\% IC $=0.23-0.65) .{ }^{51}$

Un análisis retrospectivo y no aleatorizado de los datos del estudio OPTIMAAL, evaluó la eficacia de la combinación de un beta-bloqueador más una estatina en 5477 pacientes que presentaban ICC post infarto de miocardio. Cada una de las modalidades de tratamiento estuvo asociada a una reducción significativa de la mortalidad por cualquier causa, con una disminución del riesgo relativo del $48.3 \%$ cuando ambos fármacos se utilizaban conjuntamente. ${ }^{52}$ De manera similar, un análisis retrospectivo del estudio CIBIS-II, que demostró la eficacia del uso del bisoprolol y una estatina, mostró que esta combinación confería una sobrevida mayor en comparación con aquellos individuos aleatorizados a solo una de estas drogas, generando una reducción del riesgo relativo del 96\% del evento primario. (HR: 0.14 , IC 95\%: $0.03-0.60) .^{53}$

El escrutinio post hoc de los datos del estudio Val-HeFT, evidenció que el 34\% de los 5010 pacientes investigados utilizó estatinas, a la vez que se comprobó que tenían un menor riesgo de mortalidad (HR 0.81, 95\% CI $=0.70-0.94$ ) en los 1.9 años de seguimiento promedio del estudio, siendo los más beneficiados quienes presentaban ICC..$^{54}$

Nuevamente, la falta de causalidad es un impedimento para atribuir estos resultados al uso de un determinado fármaco. Es evidente que tales estudios son de baja evidencia clínica y que cuentan con muchas limitantes estadísticas, pero permiten sentar las bases para la introducción y elaboración de estudios de mayor evidencia.

\section{Estudios prospectivos del uso de estatinas en pacientes con ICC}

En 2003 surgió un pequeño estudio prospectivo y aleatorizado de 51 pacientes con cardiomiopatía dilatada de etiología no isquémica, clase funcional NYHA III y IV, en el cual, el tratamiento con simvastatina $5 \mathrm{mg}$ /día por un mes y luego $10 \mathrm{mg} /$ día, mostró, durante el seguimiento de 14 semanas, un mejoramiento de la clase funcional de los asignados a simvastatina, asociado a un discreto aumento de la FE y a una disminución de ciertos marcadores inflamatorios. ${ }^{35}$

En una investigación llevada a cabo en pacientes con una FE menor al $40 \%$, isquémica o no (estudio UNIVERSE); se comparó el uso de rosuvastatina versus placebo. En este análisis se demostró que aquellos sujetos que ingirieron rosuvastatina, no presentaron cambios significativos en la fracción de eyección ventricular, ni tampoco en la morbimortalidad durante el periodo de seguimiento (6 meses). ${ }^{55}$

Más recientemente, en un análisis de subgrupos del estudio TNT, se comprobó que altas dosis de atorvastatina (80mg/día) eran más eficaces que las dosis usuales $(10 \mathrm{mg} /$ día) para reducir la tasa de hospitalizaciones por ICC en pacientes con enfermedad arterial coronaria, en presencia o ausencia del diagnóstico de insuficiencia cardiaca al inicio del estudio. Los autores del estudio mencionan que por cada miligramo por decilitro que descendieron los niveles de LDL colesterol, el riesgo de hospitalización se redujo en un $0.6 \%{ }^{56}$ Se reportó además una reducción del riesgo absoluto de hospitalización por ICC del $6.7 \%$, en el subgrupo de pacientes que presentaban este diagnóstico y consumieron dosis altas de atorvastatina.

El primer estudio aleatorizado y prospectivo para demostrar el efecto en la sobrevida de pacientes con ICC (en estadios NYHA II a IV) con el uso de una estatina, fue llevado a cabo en 5011 pacientes mayores de 60 años, la mayoría por etiología isquémica, quienes fueron analizados durante 32.8 meses en promedio. En el estudio no se produjo una reducción significativa en el objetivo primario (muerte de causa cardiovascular, infarto de miocardio no fatal y evento cerebrovascular no fatal), aun cuando se disminuyeron las concentraciones de la proteína $\mathrm{C}$ reactiva. El estudio por separado de los puntos secundarios, demostró una reducción 
significativa en la tasa de hospitalizaciones de causa cardiovascular en el grupo tratado con rosuvastatina (10 $\mathrm{mg}$ ), en comparación con el grupo placebo. Cabe señalar que en este estudio efectuado en pacientes mayores, no hubo diferencias estadísticamente significativas en efectos adversos entre el grupo tratado con rosuvastatina y el grupo control. ${ }^{57}$

Hace poco se publicó en el sitio de Internet de The Lancet, el segundo estudio prospectivo y aleatorizado que evaluó, como punto primario compuesto, el tiempo de sobrevida global y el tiempo de sobrevida o admisión hospitalaria por causa cardiovascular en pacientes con ICC de diversa etiología. Los sujetos estudiados se aleatorizaron en 2 grupos para recibir $10 \mathrm{mg}$ de rosuvastatina o placebo. ${ }^{58-59}$ En contraste con el estudio CORONA, el diseño del ensayo GISSI-HF analizó a 4631 pacientes mayores de 18 años con ICC, independientemente de la FE presentada (alrededor del 10\% de los pacientes tenía una $\mathrm{FE}<40 \%$ ), y se siguieron por un periodo promedio de 3.9 años. Los resultados demostraron que no existían diferencias estadísticamente significativas entre ambos brazos del estudio en el punto final primario compuesto, a pesar de la disminución del LDL-colesterol y de la proteína $\mathrm{C}$ reactiva en el grupo que recibió la estatina. Este resultado se presentó uniformemente en todos los subgrupos analizados y tampoco mostró diferencias significativas en los puntos secundarios evaluados (mortalidad cardiovascular, admisión hospitalaria por causa cardiovascular o no, muerte súbita cardíaca, infarto de miocardio y eventos cerebrovasculares). ${ }^{59}$

\section{Riesgos potenciales de la reducción del colesterol en pacientes con ICC}

Existen teorías que señalan un potencial efecto adverso de las estatinas en el contexto de la ICC; uno de ellos es la reducción de la coenzima ubiquinona, una molécula mitocondrial requerida en la fosforilación oxidativa. Se postula que este decremento puede generar un agotamiento de las reservas del adenosín-trifosfato (ATP) del miocito cardiaco y una disminución de agentes antioxidantes. ${ }^{60-63}$

Otra hipótesis propuesta es la teoría "endotoxinalipoproteína", que argumenta que al disminuirse las concentraciones de colesterol sérico, se reduce la capacidad amortiguadora que los lípidos plasmáticos poseen sobre el lipopolisacárido bacteriano, y se facilita una respuesta inflamatoria sistémica con liberación de citoquinas promotoras de la progresión de la insuficiencia cardiaca. ${ }^{60}$

Finalmente, se ha señalado cierta asociación inversa entre la mortalidad de pacientes con ICC y las concentraciones séricas de colesterol. ${ }^{64-67}$ No obstante, cabe resaltar que se trata de estudios observacionales, descriptivos, de cohorte y no intervencionistas, los cuales, por la naturaleza del análisis estadístico, no necesariamente prueban causalidad. En uno de ellos, ${ }^{64}$ los pacientes con concentraciones de colesterol más bajas, presentaban desde el inicio del periodo de seguimiento, un estadio NYHA mayor que el resto de individuos estudiados. Por otra parte, el estudio de Horwich et al ${ }^{65}$ comparó 2 muestras de pacientes de tamaño distinto ( $n=595$ en el grupo de colesterol $<160 \mathrm{mg} / \mathrm{dL}$ vrs $\mathrm{n}=10968$ en el grupo de colesterol $>160 \mathrm{mg} / \mathrm{dL}$ ), y no mostró diferencias estadísticamente significativas en la mortalidad cardiovascular entre ambos grupos.

Asimismo, los 2 estudios prospectivos publicados a la fecha $^{57,59}$ mostraron perfiles de seguridad similares entre el grupo de tratamiento y el grupo control, lo cual supone que el efecto clínico de las teorías señaladas es bajo.

\section{¿Por qué la discrepancia entre los efectos farmacológicos y clínicos?}

Surge la interrogante sobre por qué no se logra reducir en forma consistente la mortalidad en la ICC con el uso de estatinas, cuando este grupo de fármacos ha demostrado reducir eventos cardiovasculares tanto en prevención primaria como secundaria. Quizás la respuesta yace en que la mayoría de las muertes por ICC son causadas por arritmias fatales y no secundarias a un evento aterotrombótico. ${ }^{6}$ Además, las aseveraciones sobre la eficacia potencial de las estatinas en pacientes con ICC fueron hechas con base en los hallazgos de estudios observacionales o post hoc, los cuales, tal como se ha mencionado, no necesariamente prueban causalidad entre los grupos expuestos y no expuestos a un fármaco.

De forma similar, la reducción de marcadores inflamatorios demostrada en 2 de los estudios, en los cuales no se evidenció eficacia alguna en la prevención de eventos clínicos con el uso de rosuvastatina, sugiere que la actividad inflamatoria presente en la ICC indica un pequeño aspecto de la fisiopatología global de dicho síndrome, lo cual es apoyado por varios estudios que señalan la ineficacia de algunos fármacos bloqueadores de citoquinas empleados en estos pacientes ${ }^{68}$; así como una sobreestimación de los efectos pleiotrópicos de las estatinas.

\section{Conclusiones}

Si bien existen bases teóricas y efectos farmacológicos que promueven el uso de las estatinas en los pacientes con ICC, es claro que con el nivel de evidencia médica actual, sigue sin demostrarse un efecto consistente sobre la mortalidad de los pacientes con esta condición y que utilizan tales fármacos. ${ }^{69}$

De hecho, los 2 únicos estudios prospectivos $\mathrm{y}$ aleatorizados publicados a la fecha, que utilizan como indicación de tratamiento la presencia de ICC, sin considerar las concentraciones séricas de colesterol, no demostraron una reducción significativa del objetivo primario compuesto. ${ }^{57,59}$ Este hallazgo hace que veamos con cautela 
la frecuente discrepancia de los efectos farmacológicos con los efectos clínicos, así como la divergencia entre la efectividad señalada por un estudio retrospectivo, de cohortes o de subgrupos, y la de un análisis prospectivo, dirigido a la eficacia de un determinado tratamiento en un contexto específico.

A pesar de lo expuesto en esta revisión, vale la pena referirse a la abundante cantidad de evidencia clínica actual que apoya el uso de estos fármacos en la prevención primaria o secundaria de eventos cardiovasculares, y que ha logrado reducir la morbimortalidad de un grupo considerable de pacientes evaluados en diversas condiciones clínicas. ${ }^{1,2,70-71}$ Nuevamente, la publicación de más ensayos clínicos que puedan generarse en los próximos años, será la guía para el uso racionado de las estatinas en la ICC.

\section{Referencias}

1. Plehn J, Davis B, Sacks F, Rouleau J, Pfeffer M, Bernstein V. Reduction of stroke incidence after myocardial infartion with pravastatin: The cholesterol and recurrent events (CARE) study. Circulation. 1999; 99: 216-223.

2. Heart Protection Study Collaborative Group. MRC / BHF Heart Protection Study of cholesterol lowering with simvastatin in 20536 high - risk individuals: a randomized placebo - controlled trial. Lancet. 2002; 360: 7-22.

3. Raina A, Pickerin T, Shimbo D. Statin use in heart failure: A cause of concern? Am Heart J. 2006; 152:39-49.

4. Aronow W. Epidemiology, Pathophysiology Prognosis and Treatment of Systolic and Diastolic Heart Failure. Cardiol Rev. 2006; 14:108124.

5. Owan TE, Hodge DO, Herges RM, Jacobsen SJ, Roger VL, Redfield MM. Trends in prevalence and outcome of heart failure with preserved ejection fraction. N Engl J Med. 2006; 355:251-259

6. Ulate-Montero G, Ulate-Campos A. Actualización en los mecanismos fisiopatológicos de la insuficiencia cardiaca. Act Med Costarric. 2008; 50: 5-12.

7. Hoshijima M, Sah V, Wang Y, Chien K, Heller J. The low molecular weight GTPase rho regulates myofibril formation and organization in neonatal rat ventricular myocites. Biol Chem. 1998; 273:7725-7730.

8. Wettschureck N, Offermanns S. Mammalian G proteins and their cell type specific functions. Physiol Rev. 2005; 85:1159-1204.

9. Satoh M, Ogita H, Takeshita K, et al. Requirement of Rac1 in the development of cardiac hypertrophy. PNAS. 2006; 103:7432-7437.

10. Auki H, Izumo S, Sadoshima J. Angiotensin II activates RhoA in cardiac myocites. Circ Res. 1998; 82:666-676.

11. Satoh S, Ueda Y, Koyanagui M, Kadokami T, Sugano M, Yoshikawa Y, Makino N. Chronic inhibition of Rho Kinase blunts the process of left ventricular hypertrophy leading to cardiac conctractile dysfunction in hypertensive - induced heart failure. J Mol Cell Cardiol. 2003; 35:59-70.

12. Frey N, Katus H, Olson E, et al. Hypertrophy of the heart. Circulation 2004; 109:1580-1589.

13. Pimentel D, Amin J, Xiao L, Miller T, Viereck J, Olivier-Krasinski J, et al. Reactive Oxygen Species mediate amplitud-dependant hypertrophic and apoptotic responses to mechanical stretch in cardiac myocites. Circ Res. 2001; 89:453-460.
14. Nojiri H, Shimizu T, Funakoshi M, Yamaguchi O, Zhou H, Kawakami S. Oxidative Stress causes heart failure with impaired mithocondrial respiration. J Biol Chem. 2006; 281:33789-33801.

15. Liu H, Colavitti R, Rovira I, Finkel T. Redox-Dependant transcriptional regulation. Circ Res. 2005; 97:967-974.

16. Giordano F. Oxygen, oxidative stress, hypoxia, and heart failure. J Clin Invest. 2005; 115:500-508.

17. Cheng TH, Cheng PY, Shih NL, Chen IB, Ling D, Chen JJ. Involvement of reactive oxygen species in angiotensin II - induced endothelin - 1 gene expression in rat cardiac fibroblasts. J Am Coll Cardiol. 2003; 42:1845-1854.

18. Takimoto E, Kass D. Role of oxidative stress in cardiac hypertrophy and remodeling Hypertension. 2007; 49:241-248.

19. Endemann D, Schiffrin E. Endothelial dysfunction. J Am Soc Nephrol. 2004; 15:1983-1992.

20. Taniyama Y, Griendling K. Reactive Oxygen Species in the Vasculature. Hypertension. 2003; 42:1075-1081.

21. López A, Casado S. Heart Failure, redox alterations, and endothelial dysfunction. Hypertension. 2001; 38:1400-1405.

22. Ramos-Esquivel A, León-Céspedes C. Efectos no hipolipemiantes de las estatinas. Act Med Costarric. 2007; 49: 182-189.

23. Liao JK. Isoprenoids as mediators of the biological effects of statins. $\mathrm{J}$ Clin Invest. 2002; 110:285-288.

24. Takemoto M, Node K, Nakagami H, Liao Y, Grimm M, Takemoto Y, et al. Statins as antioxidant therapy for preventing cardiac myocite hypertrophy. J Clin Invest. 2001; 108:1429-1437.

25. Haendeler J, Hoffman J, Zeiher A, Dimmeler S. Antioxidant effect of statins via S-nitrosylation and activation of thioredoxin in endothelial cells. Circulation. 2004; 110:856-861.

26. Zhang H, Schmeiber A, Garlichs C, Plötze K, Damme U, Mügge A, et al. Angiotensin II-induced superoxide anion generation in human vascular endothelial cells: Role of membrane-bound NADH-/ NADPH-oxidases. Cardiovasc Res. 1999; 44: 215-222.

27. Wassmann S, Laufs U, Bäumer A, Müller K, Konkol C, Sauer H et al. Inhibition of geranylgeranylation reduces angiotensin II-mediated free radical production in vascular smooth muscle cells: involvement of angiotensin AT1 receptor expression and Rac1 GTPase. Mol Pharmacol. 2001; 59:646-654.

28. Dechend R, Fiebeler A, Park JK, Müller D, Theur J, Mervaala E, et al. Amelioration of angiotensin II - induced cardiac injury by a 3hydroxy-3-methyglutaryl coenzyme A reductase inhibitor. Circulation. 2001; 104:576-581.

29. Pliquett R, Cornish K, Zucker I. Statin therapy restores sympathicovagal balance in experimental heart failure. J Appl Physiol. 2003; 95:700704.

30. Pliquett R, Cornish K, Peuler JD. Simvastatin normalizes autonomic neural control in experimental heart failure. Circulation. 2003; 107:2493-2498.

31. Gao L, Wang W, Li YL, Shultz HD, Liu D, Cornish KG, et al. Simvastatin therapy normalizes sympathetic neural control in experimental heart failure. Circulation. 2005; 112:1763-1770.

32. Tousoulis D, Antoniades C, Bosinakou E, Kotsopoulou M, Pitsavos $\mathrm{C}$, Vlachopoulos $\mathrm{C}$, et al. Effects of atorvastatin on reactive hyperemia and inflammatory process in patients with congestive heart failure. Atherosclerosis. 2005; 178:359 - 63.

33. Sola S, Mir M, Lerakis S, Tandon N, Khan B. Atorvastatin improves left ventricular systolic function and serum markers of inflammation in nonischemic heart failure. J Am Coll Cardiol. 2006; 47:332-337.

34. May H, Muhlestein J, Carlquist J, Horne B, Campbell, Kfoury A, et al. Relation of serum total cholesterol, c-reactive protein leveles and statin therapy to survival in heart failure. Am J Cardiol. 2006; 98:653658. 
35. Node K, Fujita M, Kitakaze M, Hori M, Liao J. Short-term statin therapy improves cardiac function and symptoms in patients with idiopathic dilated cardiomyopathy. Circulation. 2003;108:839-843.

36. Strey $\mathrm{CH}$, Young JM, Lainchbury JH, Frampton C, Nicholls M, Richards A, et al. Short term statin treatment improves endothelial function and neurohormonal imbalance in normocholesterolaemic patients with non - ischemic heart failure. Heart. 2006; 92:16031609.

37. Schäfer A, Fraccarollo D, Eigenthaler M, Tas P, Firnschild A, Frantz A, et al. Rosuvastatin reduces platelet activation in heart failure. Arterioscler Thromb Vasc Biol. 2005; 25:1071-1077.

38. Horwich T, Middlekauff $H$. Potential autonomic nervous system effects of statins in heart failure. Heart Fail Clin. 2008; 4: 163-170.

39. Tousoulis D, Antoniades C, Bosinakou E, Kotsopoulou M, Tsioufis C, Tentolouris $\mathrm{C}$, et al. Effects of atorvastatin on reactive hyperaemia and the thrombosis - fibrinolysis system in patients with heart failure. Heart. 2005; 91:27-31.

40. Strey CH, Young JM, Lainchbury JH, Frampton CM, Nicholls MG, Richards AM, et al. Short-term statin treatment improves endothelial function and neurohormonal imbalance in normocholesterolaemic patients with non-ischaemic heart failure. Heart. 2006; 92:16031609.

41. Shanes JG, Minadeo KN, Moret A, Grover M, Tabaie SA. Statin therapy in heart failure: Prognostic effects and potential mechanisms. Am Heart J. 2007; 154: 617-623.

42. Bauersachs J, Galuppo P, Fraccarollo D, Christ M, Ertl G. Improvement of left ventricular remodeling and function by hydroxymethylglutaryl coenzyme A reductase inhibition with cerivastatin in rats with heart failure after myocardial infarction. Circulation. 2001; 104:982-985

43. Senthil V, Chen S, Tsybouleva N, Halder T, Naqueh SF, Willerson J, et al. Prevention of cardiac hypertrophy by atorvastatin in a transgenic rabbit model of human hypertrophic cardiomyopathy. Circ Res. 2005; 97: 285-292.

44. KjesKshus J, Pedersen TR, Olsson AG, Faergeman A, Pyörälä K. The effects of simvastatin on the incidence of heart failure in patients with coronay heart disease. J Card Fail. 1997; 3:255-256.

45. Sacks FM, Pfeffer MA, Moye LA, Rouleau J, Rutherford J, Cole TG, et al. The effect of pravastatin on coronary events after myocardial infarction in patients with average cholesterol levels. Cholesterol and Recurrent Events Trial investigators. N Engl J Med.1996;335:10011009.

46. Horwich T, MacLellan WR, Fonarow G. Statin therapy is associated with improved survival in ischemic and non-ischemic heart failure. J Am Coll Cardiol. 2004; 43:642-648.

47. Ray J, Gong Y, Sykora K. Statin use and survival outcomes in elderly patients with heart failure. Arch Intern Med. 2005; 165:62-67.

48. Go A, Lee W, Yang J, Lo J, Gurwitz J. Statin therapy and risks for death and hospitalization in chronic heart failure. JAMA. 2006; 296:2105-2111.

49. Foody JM, Shah R, Galusha D, Masoudi FA, Havranek EP, Krumholz HM. Statins and mortality among elderly patients hospitalizad with heart failure. Circulation. 2006; 113:1086-1092.

50. Ezekowitz J, McAlister F, Humphries K, Norris C, Tonelli M, Ghali $\mathrm{W}$, et al. The association among renal insufficiency, pharmacotherapy, and outcomes in 6,427 patients with heart failure and coronary artery disease. J Am Coll Cardiol. 2004; 44: 1587-192.

51. Mozaffarian D, Nye R, Levy W. Statin therapy is associated with lower mortality among patients with severe heart failure. Am J Cardiol. 2004; 93:1124-1129.

52. Hognestad A., Dickstein K., Myhre E. Snappin S, Kjekshus J. OPTIMAAL Investigators. Effect of combined statin and beta-blocker treatment on one-year morbidity and mortality after acute myocardial infarction associated with heart failure. Am J Cardiol. 2004; 93. 603606.
53. Krum H, Bailey M, Meyer W, Verkenne P, Dargie H, Lechat P, et al. Impact of statin therapy on clinical outcomes in chronic heart failure patients according to beta-blocker use: results of CIBIS II. Cardiology. 2007; 108:28-34

54. Krum H, Caretta E, Latini R, Maggioni AP, Anand I, Masson S, et al. Statins and symptomatic chronic systolic heart failure: a post-hoc analysis of 5010 patients enrolled in Val-HeFT. Int J Cardiol. 2007; 119:48-53.

55. Krum H, Ashton E, Reid C, Kalff V, Roger J, Amarena J, et al. Doubleblind, randomized, placebo-controlled study of high-dose HMG CoA reductase inhibitor therapy on ventricular remodeling, proinflammatory cytokines and neurohormonal parameters in patients with chronic systolic heart failure. J Card Fail. 2007; 13: 1-7.

56. Khush K, Waters D, Bittner D, Deedwania PC, Kastelein JJ, Lewis SJ, et al. Effect of high-dose atorvastatin on hospitalizations for heart failure. Subgroup analysis of the treating to new targets (TNT) study. Circulation. 2007; 115:576-583.

57. Kjieshus J, Apetrei E, Barrios V, Böhm M, Cleland J, Cornel J, et al. Rosuvastatin for older patients with systolic heart failure. N Engl J Med. 2007; 357:1-14.

58. Tavazzi L, Tognoni G, Franzosi MG, Latini R, Maggioni A, Marchioli $\mathrm{R}$, et al.. Rationale and design of the GISSI heart failure trial: a large trial to assess the effects of n-3 polyunsaturated fatty acids and rosuvastatin in symptomatic congestive heart failure. Eur J Heart Fail. 2004; 6:635-641.

59. GISSI-HF investigators. Effects of rosuvastatin in patients with chronic heart failure (the GISSI-HF trial): a randomized, double-blind, placebo-controlled trial. Lancet. En: http://download.thelancet.com/ pdfs/journals/0140-6736/PIIS0140673608612404.pdf Consultado el 15 de setiembre de 2008 .

60. Rauchhuss M, Coats AJ, Anker S. The endotoxin-lipoprotein hypothesis. Lancet. 2000; 356: 930-933.

61. Littarru GP, Langsjoen P. Coenzyme Q10 and statins: biochemical and clinical implications. Mithocondrion. 2007; S168-S174.

62. De Pinieux G. Lipid-lowering drugs and mithochondrial function: effects of HMG-CoA reductase inhibitors on serum ubiquinone and blood lactate/pyruvate ratio. Br J Clin Pharmacol. 1996; 42:333-337.

63. van der Harst P, Voors A, van Gilst WH, Böhm M, van Veldhussein DJ. Statin in the treatment of chronic heart failure: biological and clinical consideration. Cardiovas Res. 2006; 443-454.

64. Afsarmanesh N, Horwich TB, Fonarow GC. Total cholesterol levels and mortality risk in nonischemic systolic heart failure. Am Heart J. 2006; 152; 1077-1083.

65. Horwich TB, Hamilton MA, Maclellan WR, Fonarow GC. Low serum total cholesterol is associated with marked increase in mortality in advanced heart failure. J Card Fail. 2002; 8: 216-224.

66. Rauchhaus M, Clark AL, Doehner W, Davos C, Bolger A, Sharma R, et al. The relationship between cholesterol and survival in patients with chronic heart failure. J Am Coll Cardiol. 2003; 42: 1933-1940.

67. Ramasubbu K, Mann D. The emerging role of statins in the treatment of heart failure. J Am Coll Cardiol. 2006; 47: 342-344.

68. Mann D, McMurray J, Packer M, Swedberg K, Borer J, Colucci W, et al. Targeted anticytokine therapy in patients with chronic heart failure: results of the randomized etanercept worldwide evaluation. Circulation. 2004; 109: 1594-1602.

69. Ramasubbu K, Mann D. The emerging role of statins in the treatment of heart failure. J Am Coll Cardiol. 2006; 47: 342-344.

70. LIPID Study Group. Prevention of cardiovascular events with pravastatin in patients with coronary heart disease and a broad range of initial cholesterol levels. N Engl J Med. 1998; 339: 1349-1357.

71. Downs JR, Clearfield M, Weis S, Whitney E, Shapiro D, Beere PA, et al. Primary prevention of acute coronary events with lovastatin in men and women with average cholesterol levels. JAMA. 1998; 279: 16151622 . 